\title{
Rapid Coating of Ultraviolet Shielding Colloidal Crystals
}

\author{
Likun Wang ${ }^{1,2}, \mathrm{Yu} \mathrm{Xu}^{3}$, Zhaoran Chu ${ }^{4}$, Wenwei Tang ${ }^{5}$, Yanfei Qiu ${ }^{4}$, Xueling Zhao ${ }^{4}$, \\ Weizhong Jiang $1,2, *$, Jiayi Ye ${ }^{2,6, *}$ and Cheng Chen $4,7, * \mathbb{D}$ \\ 1 College of Materials Science and Engineering, Donghua University, Shanghai 201620, China; \\ flowerangel826@aliyun.com \\ 2 China National Inspection \& Testing Centre for Ophthalmic Optic Glass and Enamel Products, \\ Donghua University, Shanghai 201620, China \\ 3 Aerospace System Engineering Shanghai, Shanghai 201108, China; xuyu805casc@163.com \\ 4 School of Environmental and Materials Engineering, College of Engineering, \\ Shanghai Polytechnic University, Shanghai 201209, China; chuzhaoran01@163.com (Z.C.); \\ dailyqyf191021@163.com (Y.Q.); xlzhao@sspu.edu.cn (X.Z.) \\ 5 Modern Service Department, College of International Vocational Education, \\ Shanghai Polytechnic University, Shanghai 201209, China; tangww@sspu.edu.cn \\ 6 Research Institute, Donghua University, Shanghai 201620, China \\ 7 Research Center of Resource Recycling Science and Engineering, Shanghai Polytechnic University, \\ Shanghai 201209, China \\ * Correspondence: jwzh@dhu.edu.cn (W.J.); jiayiye@163.com (J.Y.); chencheng@sspu.edu.cn (C.C.)
}

Received: 27 April 2020; Accepted: 8 June 2020; Published: 12 June 2020

\begin{abstract}
A facile spray coating preparation of ultraviolet (UV) shielding Poly(methyl methacrylate) (PMMA) based colloidal photonic crystal (PC) films was presented, where the UV radiation was physically resisted by the periodic structure. The specific wavelength within the UV regime could be tuned as required by varying the size of the monodispersed PMMA colloids. Such crystal coatings could be rapidly prepared in optical glasses with controllable thickness of $\sim 5 \mu \mathrm{m}$, which could simultaneously resist UV-254 with the efficiency of $77.43 \%$. The monochromaticity of the crystal coatings ensures their potential in UV shielding materials of direct physical skin contact type.
\end{abstract}

Keywords: colloidal crystal; photonic crystal; UV shielding; coating; self-assembly

\section{Introduction}

Photonic crystals (PCs) are long-term ordered structures on a nano/micro scale. Their periodic dielectric structures can selectively modulate electromagnetic waves of certain frequencies, and the forbidden frequencies are called photonic band gaps (PBGs) [1,2]. Currently, functional photonic crystal materials, such as photonic fiber, membrane, ink, paper and sensor materials, have been developed. One of the most facile methods for the preparation of photonic crystals is the self-assembly of so-called colloidal crystals (CCs) using nano-colloids [3,4]. Many methods for the preparation of CCs have been developed, including spin coating [5], deposition [6-8] and methods based on induced self-assembly [9-11]. The CC assemblies can be either closely packed (CP) or non-closely packed (NCP) periodic face-centered cubic (FCC) structures, under gravitational, electrostatic or magnetic forces. Each colloidal particle can be considered a lattice of the crystal, whose diffraction wavelength (i.e., PBG) can be calculated according to Bragg's law:

$$
m \lambda_{0}=2 n_{\mathrm{a}} d_{111} \operatorname{Sin} \theta,
$$


where $m$ is the order of diffraction, $\lambda_{0}$ is the diffracted wavelength in air, $n_{\mathrm{a}}$ is the average refractive index of the system, $d_{111}$ is the interplanar crystal spacing, and $\theta$ is the observation angle between the incident light and the normal $\left(\theta=90^{\circ}\right.$ during detection).

Since CCs are formed by spontaneous arrangement, this mesa-stable structure is usually fragile due to the weak interparticle force between colloids. In our previous work, a series of PC materials were prepared using polymer hydrogels to stabilize CC composites [12-14]. We also proposed a simple method to fabricate a large area of ordered and robust ultrathin colloidal photonic film material that can effectively diffract monochromatic light [15]. By controlling the size of the nano-colloids, the diffraction color can be adjusted from ultraviolet (UV) to visible light. However, this method is only applicable in coating the crystal layer on a substrate with a flat surface, which limits its implementation. It has been reported that colloidal particles can be rapidly assembled on the surface of various materials by spraying coating [16]. Hence, we designed a spraying method to form three-dimensional (3D) CCs on non-flat surfaces, such as the optical lens, which is expected to be used for physical UV protection, and provide a method for the fabrication of a multilevel structure of PC materials [17].

UV radiation is harmful to lots of materials, especially organic materials, and the human body also needs UV shielding to protect vital organs, such as the skin and eyes. The core idea of this work is to modulate and combine PBGs of CC materials in the appropriate regime to achieve UV shielding, while maintaining high transparency of the materials. To this end, we did the following work: (1) selection of polymer materials, i.e., polymethyl methacrylate (PMMA) nano-colloids, with a high molecular weight, which will not penetrate the human skin; (2) dispersing a solvent of the nano-colloids, i.e., the mixture of ethanol and water, which is non-toxic and could easily be removed. Meanwhile, PMMA is a common non-toxic material with good biocompatibility, and has high transparency. Therefore, these materials were utilized to construct CC coating materials, and their application as UV protection materials when coated on the surfaces of optical glasses was also investigated.

\section{Materials and Methods}

\subsection{Materials}

Methyl methacrylate (MMA), acrylic acid (AA) and ammonium persulfate (APS) were purchased from Shanghai Chemical Agent Co., Ltd. All other reagents were of analytical grade and obtained from Sigma-Aldrich. Optical glasses (Essillor, $n=1.591, \mathrm{D}=75 \mathrm{~mm},+00.00$ ) were used as received. The glassware used in experiments was cleaned with RCA solution [5:1:1 mixture of water, hydrogen peroxide $(30 \%)$ and ammonia $(28 \%)]$ at $75{ }^{\circ} \mathrm{C}$ for $30 \mathrm{~min}$. Ultrapure water $(18.2 \mathrm{M} \Omega \cdot \mathrm{cm})$ was used in all experiments. All materials were used as received without further purification.

\subsection{Preparation of CC Films}

Monodisperse PMMA latexes (117 nm, 138 nm, $162 \mathrm{~nm}$ and $200 \mathrm{~nm}$ ) were prepared via the universal emulsifier-free polymerization procedure that we previous developed [18]. The preparation process was as follows: $200 \mathrm{~mL}$ ultrapure water, $20 \mathrm{~mL}$ MMA and $1.5 \mathrm{~mL}$ AA were added to the three-neck flask with oil bath heated to $100^{\circ} \mathrm{C}$, and rapid stirring was sustained at $300 \mathrm{RPM}$ to keep the system homogeneously mixed. After the system was boiled, $0.4 \mathrm{~g}$ APS was added for the initiation of the polymerization, and the mixture rapidly changed to milky white. After $0.5 \sim 1 \mathrm{~h}$ of polymerization, the reaction was ended by transferring the flask into ice bath. After the resulting emulsion was cooled to room temperature, the PMMA colloid emulsion was dialyzed with ultrapure water for 7 to 14 days, to remove unreacted organic molecules and ionic impurities. The purified colloids were dried and dispersed in ethanol/water (1/3) solvent and stored in the glass jar. The ethanol/water solvent could facilitate the dispersing of the PMMA colloids to form homogeneous suspension. The PMMA latex containing $10 \%$ colloids was added to the spray gun container, and the air pressure was adjusted to 2 bars, the flow rate was $2 \mathrm{~m} / \mathrm{min}$, and the spray was carried out at a distance of $10 \mathrm{~cm}$ from the substrate (optical glasses). The experimental method is shown in Figure 1. 

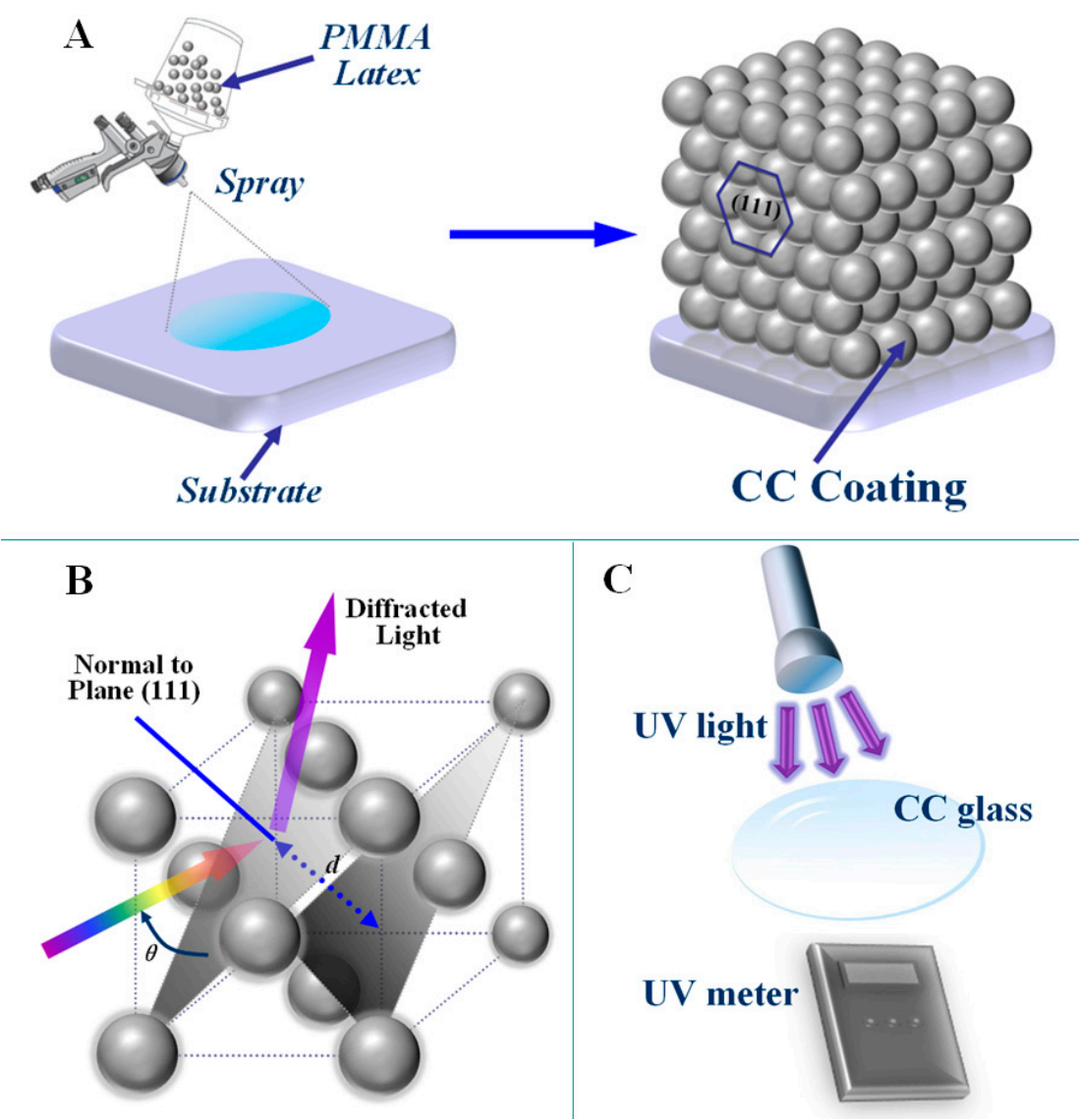

Figure 1. Illustration of the: (A) construction of PMMA CC; (B) Bragg diffraction of the CC and (C) UV shielding test of CC coated glasses.

\subsection{The Characterization of PC Films}

Structural and morphological characterizations of the crystals were conducted using a scanning electron microscope (SEM, Hitachi, S-4800, Japan) operating at an acceleration voltage of 3-10 kV, and the samples were sputter coated with a thin layer of Au. The PBG of the crystal film was confirmed by measuring the reflection spectra and integral visible transmittance using a fiber optic spectrometer (Ocean Optics, USB 4000-XR1-ES, with DH-2000-BAL light source, Winter Park, FL, USA). Spectra were captured between the wavelengths of 200 and $800 \mathrm{~nm}$. For the UV shielding test, a deep UV light of $254 \mathrm{~nm}$ was exited and a UV photometer (LS-126, China) was detected at a fixed distance of $2 \mathrm{~cm}$. Optical photographs were taken with a digital camera (Canon, EOS 6D, Japan) with macro lens (Tamron, 272E, Japan).

\section{Results and Discussion}

The design of this experiment utilizes the spraying method to realize the rapid assembly of nano-colloids. The crucial factor is the cooperative control of the interaction between the colloids and the volatilization of the solvent [19], such that rapid and high quality stacking of the PMMA colloids could be achieved while maintaining the high transparency of the coating.

Figure 2 shows the SEM images of PMMA CCs that self-assembled via spray coating. It can be observed that PMMA colloids have good monodispersibility, that is, the particle size is uniform, and the self-assembled PMMA colloids have an ordered CP structure. Figure 2A,B show the top view and cross section of the CC sample self-assembled by 117-nm PMMA colloids, respectively. The hexagon arrangement suggested (111) the direction of the FCC structure, as each colloid was surrounded by six neighboring colloids. Interestingly, the colloids were packed and connected by small necks, which might have been formed in the process of solvent evaporation and crystal assembly, 
due to the reaction of the surface carboxyl group from the AA. On the other hand, the necks might have also resulted from the rapid assembly of the CCs. The necking formed during the spray process between neighboring colloids (also c.f. Figure S1) could enhance the mechanical strength of the PMMA CCs [20].

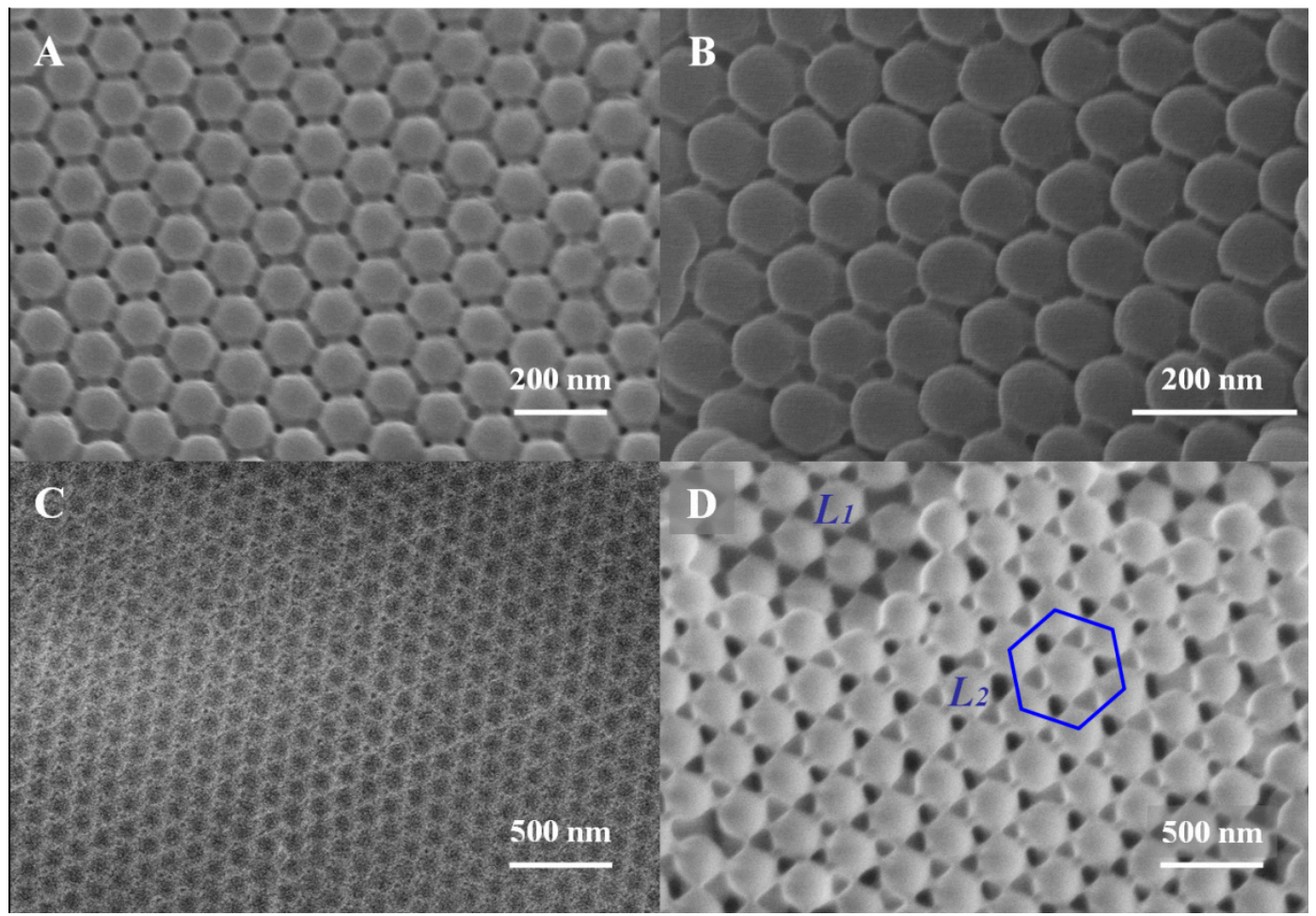

Figure 2. SEM images of PMMA self-assembled CCs: (A) Top view of 117-nm CC; (B) Cross section of 117-nm CC; (C) Large-scale top view of 117-nm CC; (D) Top view of 2-layer 200-nm CC.

Figure 2C shows the long-range ordered flat surface of the 117-nm CC formed at the liquid-air interface, which plays an important role in the Bragg diffraction properties of the photonic material. Figure 2D shows the two-layer structure formed by the assembly of 200-nm PMMA microspheres with larger particle sizes. The first layer was labeled with $L_{1}$ and the second with $L_{2}$ to guide the eye. The hexagonally ordered structure could be observed in both layers, but the sample showed a slight NCP structure with longer necking between the colloids. It is speculated that this resulted from the incomplete assembly, caused by the mismatched rate between the assembly and the solvent evaporation for the low layered crystals. The proposed self-assembly process is shown in Figure 3. In the process of the spraying assembly, the movement of the colloids was driven by the surface tension of the solvent and the repulsive force between the colloids [19]. When the droplets are thin and the layers of the crystal are low, the adhesive force of the bottom colloids is greater than that of the surface tension, resulting in larger neighboring spacing (Figures S1 and S2). As the droplet thickness increases, the volatilization of the solvent slows down, leading to the complete assembly of the upper surface colloids during solvent volatilization. Then, a secondary layer will assemble following the upper assembled layer as a template. Subsequently, the layer-by-layer assembly occurs, and the crystal can be formed as the solvent is completely removed. Therefore, the crystal with more layers has closer packing, which is consistent with the results reported [16].

The spectral characterization results of the obtained crystals are shown in Figure 4A. The PBG of the 117-nm PMMA CC coating is $\sim 258 \mathrm{~nm}$, and the full width at half max (FWHM) of the diffracted 
peak in units of wavelength is $\sim 15 \mathrm{~nm}$. According to the relationship between the interfacial spacing of the FCC and the particle size of the colloids, Equation (1) can be written as:

$$
\lambda_{0}=2 n_{a} \sqrt{\frac{2}{3}} D,
$$

where $D$ is the diameter of the colloids, and the average crystal refractive index $n_{\mathrm{a}}$ can be calculated by the following equation:

$$
n_{a}^{2}=\sum_{\mathrm{i}} n_{i}^{2} \varphi_{\mathrm{i}}
$$

where the volume factor $\left(\varphi_{\mathrm{PMMA}}=0.74\right.$ and $\left.\varphi_{\text {air }}=0.26\right)$, refractive index $\left(n_{\mathrm{PMMA}}=1.49\right.$ and $\left.n_{\text {air }}=1\right)$. Therefore, the calculated $n_{\mathrm{a}}=1.379$. Thus, Equation (2) can be rewritten as:

$$
\lambda_{0}=2.252 D,
$$

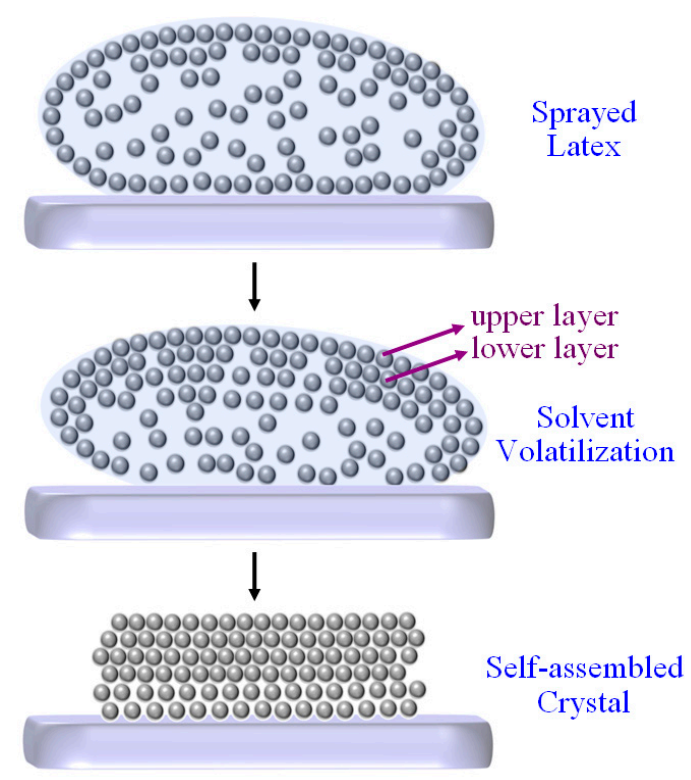

Figure 3. Schematic diagram of the self-assembly process of sprayed PMMA colloids.
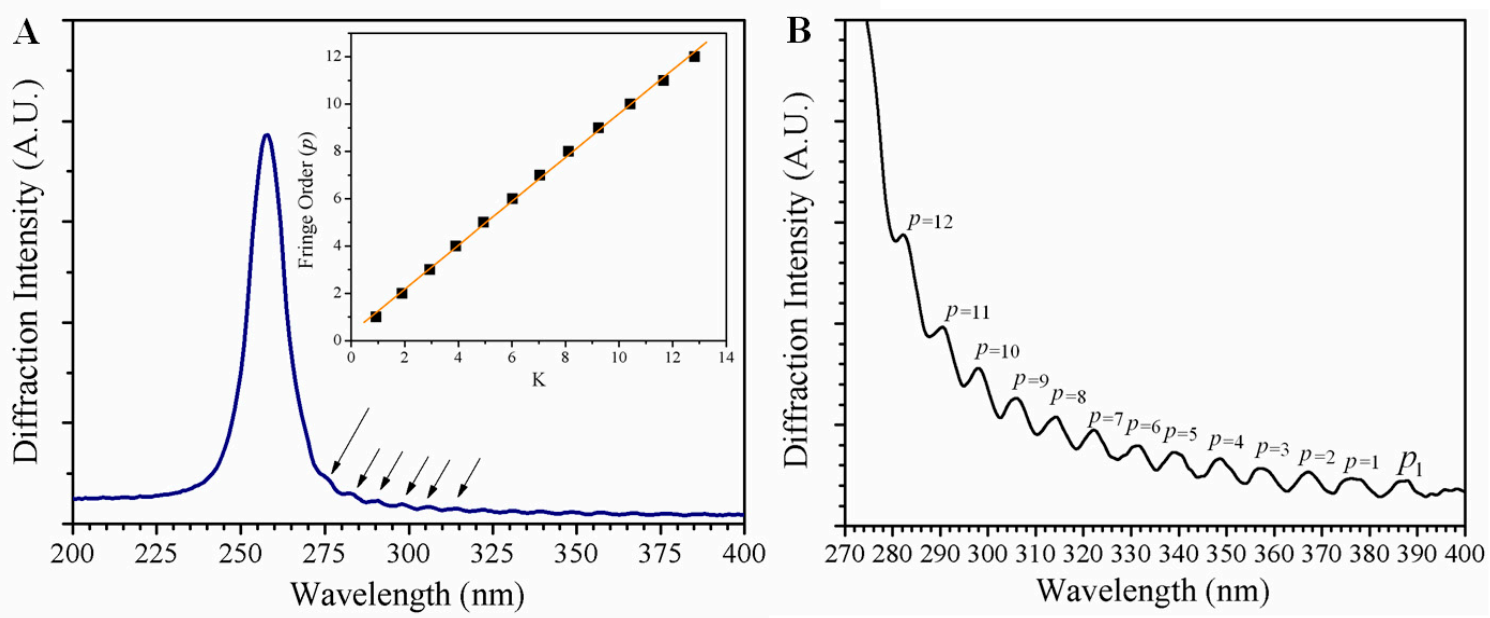

Figure 4. (A) Diffraction spectra of PMMA assembled crystal, and the evident FP fringes are labeled by arrows (Inset: FP fringe plot); (B) An enlarged view of the FP interference fringes. 
According to the above equation, the theoretical diffraction peak of the 117-nm PMMA CC is $263.5 \mathrm{~nm}$, while the detected diffraction peak is located at $258 \mathrm{~nm}$, which has a blueshift of $\sim 5.5 \mathrm{~nm}$ from the theoretical calculation. Then, the effective refractive index $n_{\mathrm{a}}$ value is calculated as 1.350 , and the overall packing factor of the PMMA is calculated as 0.72 , which is close to the result reported elsewhere [20].

It can be observed from the diffraction spectra that several additional weak peaks appeared at the side of the most intense peak of $258 \mathrm{~nm}$. Such regularly spread peaks are called Fabry-Pérot fringes (FP), and are due to isoclinic interference, which arises from multiple beam interference, with light reflected between the top and bottom faces of the crystal. FP is a non-destructive method for the thickness measurement of thin films; the fewer the colloids layers within the crystal film, the broader the FP interference fringes, with a looser distribution. On the contrary, the weaker the interference peak, with the greater the crystal's thickness, and the smaller the spacing between the FP fringes, until they disappear [21]. Generally, the FP fringe occurs as the crystal sample has flat top and bottom faces with uniform thicknesses, which are usually less than $8 \mu \mathrm{m}$. Under the condition of the vertical incidence of the detection light source, if the maximum wavelength that can be detected after crystal interference is $\lambda_{1}$, then:

$$
p_{1} \lambda_{1}=2 n_{a} T
$$

where $p_{1}$ is an integer, $T$ is the thickness of the crystal film, and the wavelength relation at the interference peak $p$ in the direction of the shorter interference wavelength is:

$$
\left(p+p_{1}\right) \lambda_{\mathrm{p}}=2 n_{a} T
$$

where $p$ is a positive integer, which numbers the interference fringe from $\lambda_{1}$, and $\lambda_{p}$ is the wavelength of subsequent fringes at shorter wavelengths. Combining Equations (5) and (6), the film thickness $T$ can be predicted as follows:

$$
T=\frac{p \lambda_{\mathrm{p}} \lambda_{1}}{2 n_{a}\left(\lambda_{1}-\lambda_{\mathrm{p}}\right)},
$$

Accordingly, $L$, the number of assembled layers of the crystal film, can be calculated by the ratio of the film's thickness to the crystal plane's spacing; that is:

$$
\begin{aligned}
& L=\frac{T}{d_{111}}=\frac{p \lambda_{\mathrm{p}} \lambda_{1}}{\lambda_{0}\left(\lambda_{1}-\lambda_{\mathrm{p}}\right)}, \\
& K=\frac{2 n_{a}\left(\lambda_{1}-\lambda_{\mathrm{p}}\right)}{\lambda_{\mathrm{p}} \lambda_{1}} \times 10^{4},
\end{aligned}
$$

The detectable FP fringes are labeled in Figure 4B. The longest interference fringe observable, $\lambda_{1}$, is in this case at $387 \mathrm{~nm}$, and taking the above calculated actual average refractive index $n_{\mathrm{a}}=1.350$, the correlation coefficient $K$ value of the sample can be calculated [22]. Detailed results are listed in Table S1. The thickness of crystal film is calculated as between 4680 and $5140 \mathrm{~nm}$, with an average value of $4986 \mathrm{~nm}$, that is, $~ 5 \mu \mathrm{m}$ (maximum error of $6 \%$ and average error of $3 \%$ ). The number of assembled layers is calculated as between 49 and 54 layers, and the average value is 52 layers (maximum error of $6 \%$ and average error of 3\%). Despite the possible errors caused by calculation, the PMMA crystal array sample has excellent ordering and flatness, with a reliable thickness of $\sim 5 \mu \mathrm{m}$ and $\sim 52$ layers of colloids stacking (the error analysis of $T$ and $L$ is shown in Figure S3). It can be found that the overall error of the calculation results is less than $3 \%$. Thus, the overall flatness and diffraction performance of the crystal film are comparable with that of the previously reported coating material [15,23].

The relationship between spraying time and film thickness was also analyzed (c.f. Figure S4), and a linear plot indicated that the stacking/assembling of PMMA colloids could be controlled by spraying time; thus the resulting thickness of the crystal coating could be controlled as required. 
The UV shielding performances of PMMA CCs prepared with various particle sizes were investigated, and all the CC coated samples were controlled, with $\sim 5 \mu \mathrm{m}$ in thickness, and exposed under UV irradiation at a fixed distance of $5 \mathrm{~cm}$. As listed in Table 1, all the crystal samples constructed by different PMMA colloids showed related PBGs that were consistent with calculated values, which proved the good assembling of the PMMA CCs. The PBGs could be tuned to cover the whole UV regime as needed, by using PMMA colloids with different diameters. The CC film constructed by 117-nm PMMA colloids possessed the stop-band of $258 \mathrm{~nm}$, which is compatible with the excitation wavelength of the UV light, and thus has the better resisting ability, of UV-254, than other samples. The CC coating reflected $\sim 77.43 \%$ of the UV radiation, while maintaining the transparency of the substrate glasses, which also coheres with the reflection percentage of $\sim 80 \%$ in Figure $4 \mathrm{~A}$. Considering that the electromagnetic waves with longer wavelengths could penetrate at lower angles, the angle-dependence of the Bragg diffraction actually occurred since the colloidal photonic crystal was FCC structured, and the angle-dependent propagation is unavoidable, with an acceptable percentage less than $\sim 3 \%$.

Table 1. UV-254 shielding performance of PMMA photonic crystal diffraction.

\begin{tabular}{ccccc}
\hline $\boldsymbol{D}_{\text {PMMA }}(\mathbf{n m})$ & $\boldsymbol{\lambda}_{\mathbf{0}}(\mathbf{n m})$ & $\lambda_{\text {Calculated }}(\mathbf{n m})$ & UV 254 Intensity $\left(\boldsymbol{\mu w} / \mathbf{c m}^{2}\right)$ & UV Resisting Efficiency $(\%)$ \\
\hline air & - & - & 2162 & - \\
117 & 258.0 & 263.5 & 488 & 77.428 \\
138 & 312.5 & 310.8 & 1326 & 38.668 \\
162 & 366.0 & 364.8 & 1777 & 17.808 \\
200 & 456.5 (Figure S5) & 450.4 & 1922 & 11.101 \\
\hline
\end{tabular}

Considering that UV protection needs to shield UV-254 to UV-365 at the same time, three kinds of PMMA colloids were combined to produce a full UV shielding coating, as shown in Figure 5. We originally expected that a broad diffraction peak would appear over the 250 to $365 \mathrm{~nm}$ region, yet three independent but connected peaks were detected. For the purpose of whole-UV-region protection for glass, skin and other surfaces, further combinations would need to be investigated.

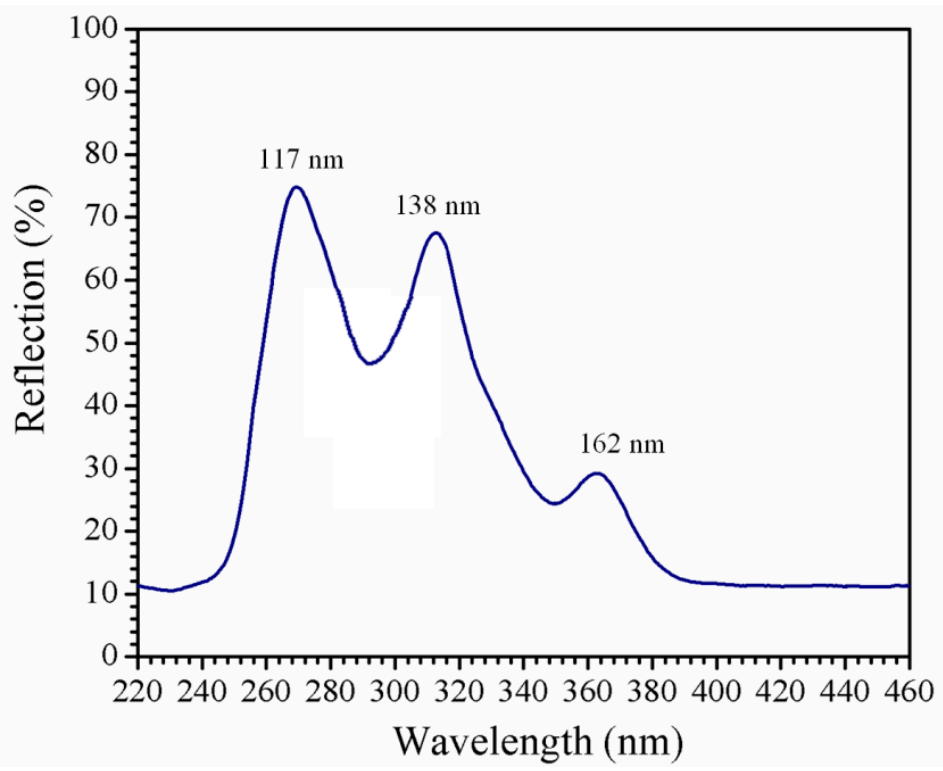

Figure 5. Diffraction property of the CC coating sample combining 117-nm, 138-nm and 162-nm PMMA colloids.

\section{Conclusions}

In summary, a facile approach to UV shielding colloidal photonic films was demonstrated, where the UV radiation was resisted by the periodic structure, without chemical reactions. 
Specific wavelengths within the UV regime could be tuned as required by varying the size of the monodispersed PMMA colloids. Such crystal coatings could be rapidly prepared on the optical glasses, that simultaneously had UV-254 resistance of $77.43 \%$. The monochromaticity of the crystal coatings ensures their potential in UV shielding materials that are in direct physical contact with skin. We also anticipate that such UV shielding system can be a promising candidate as coating material for fine surfaces and aero surfaces.

Supplementary Materials: The following are available online at http://www.mdpi.com/2073-4352/10/6/502/s1, Figure S1: Schematic representation of the motion of PMMA colloids during self-assembling, Figure S2: Stacking comparison of CC with different thickness: upper is a 2-layer assembly of 200-nm PMMA and lower is a 52-layer assembly of 117-nm PMMA, Figure S3: Thickness $(T)$ and layers $(L)$ analysis of spray coated 117-nm PMMA CC, Figure S4: Time dependence of the PMMA crystal thickness during spray process, Figure S5: 200-nm PMMA crystal coated on the optical glasses using spray process; and Table S1: Calculated data according to Fabry-Pérot fringes.

Author Contributions: The project was designed by W.T. and C.C.; L.W. and Y.Q. prepared the samples; Y.X. conducted the UV test; Z.C. collected optical data; L.W. wrote the paper under the supervision of W.J., J.Y. and C.C.; Z.C., X.Z. and C.C. revised the paper. All authors have read and agreed to the published version of the manuscript.

Funding: This research was funded by the State Key Laboratory for Modification of Chemical Fibers and Polymer Materials and China National Inspection \&Testing Centre for Ophthalmic Optic Glass and Enamel Products, the Sailing Project from Science and Technology Commission of Shanghai Municipality (17YF1406600), the Chenguang project supported by Shanghai Municipal Education Commission (18CG68), the key subject of Shanghai Polytechnic University (Material Science and Engineering, XXKZD1601, EGD19XQD03).

Conflicts of Interest: The authors declare no conflict of interest.

\section{References}

1. Yablonovitch, E. Inhibited spontaneous emission in solide-state physics and electronics. Phys. Rev. Lett. 1987, 58, 2059-2062. [CrossRef] [PubMed]

2. John, S. Strong localization of photons in certain disordered dielectric superlattices. Phys. Rev. Lett. 1987, 58, 2486-2489. [CrossRef] [PubMed]

3. Coukouma, A.E.; Asher, S.A. Increased volume responsiveness of macroporous hydrogels. Sensor. Actuat. B 2018, 255, 2900-2903. [CrossRef]

4. Maity, A.; Mujumdar, S.; Polshettiwar, V. Self-assembled photonic crystals of monodisperse dendritic fibrous nanosilica for lasing: Role of fiber density. ACS Appl. Mater. Interfaces 2018, 10, 23392-23398. [CrossRef] [PubMed]

5. Jiang, P.; McFarland, M.J. Wafer-scale periodic nanohole arrays templated from two-dimensional nonclose-packed colloidal crystals. J. Am. Chem. Soc. 2005, 127, 3710-3711. [CrossRef]

6. Meng, K.; Gao, S.S.; Wu, L.L.; Wang, G.; Liu, X.; Chen, G.; Liu, Z.; Chen, G. Two-dimensional organic-inorganic hybrid perovskite photonic films. Nano Lett. 2016, 16, 4166-4173. [CrossRef] [PubMed]

7. Wang, X.Y.; Husson, S.M.; Qian, X.H.; Wickramasinghe, R. Vertical cell assembly of colloidal crystal films with controllable thickness. Mater. Lett. 2009, 63, 1981-1983. [CrossRef]

8. Li, C.; Hong, G.S.; Qi, L.M. Nanosphere lithography at the gas/liquid interface: A general approach toward free-standing high-quality nanonets. Chem. Mater. 2010, 22, 476-481. [CrossRef]

9. Chen, K.; Fu, Q.Q.; Ye, S.Y.; Ge, J.P. Multicolor printing using electric-field-responsive and photocurable photonic crystals. Adv. Funct. Mater. 2017, 27, 1702825. [CrossRef]

10. Chen, C.; Dong, Z.Q.; Chen, H.W.; Chen, Y.; Zhu, Z.G.; Shih, W.H. Two-dimensional photonic crystals. Prog. Chem. 2018, 30, 775-784.

11. Wang, X.H.; Chen, G.; Dong, Z.Q.; Zhu, Z.G.; Chen, C. Progress in molecular imprinted photonic crystals. J. Mater. Eng. 2020, 48, 60-72. [CrossRef]

12. Chen, C.; Zhu, Y.H.; Bao, H.; Yang, X.L.; Li, C.Z. Physically controlled cross-linking in gelated crystalline colloidal array photonic crystals. ACS Appl. Mater. Interfaces 2010, 2, 1499-1504. [CrossRef] [PubMed]

13. Chen, C.; Zhu, Y.H.; Bao, H.; Zhao, P.; Jiang, H.L.; Peng, L.M.; Yang, X.L.; Li, C.Z. Solvent-assisted poly(ainyl alcohol) gelated crystalline colloidal array photonic crystals. Soft Matter 2011, 7, 915-921. [CrossRef]

14. Wang, X.H.; Qiu, Y.F.; Chen, G.; Chu, Z.R.; Shadike, A.; Chen, C.S.; Chen, C.; Zhu, Z.G. Self-healable poly(vinyl alcohol) photonic crystal hydrogel. ACS Appl. Polym. Mater. 2020, 2, 2086-2092. [CrossRef] 
15. Chen, C.; Dong, Z.Q.; Xu, Y.; Wang, X.H.; Lu, H.; Qiu, Y.F.; Zhu, Z.G. Ultrathin colloidal crystal layer as transparent photonic films. Micro Nano Lett. 2019, 14, 1-4.

16. Cui, L.Y.; Zhang, Y.Z.; Wang, J.X.; Ren, Y.B.; Song, Y.L.; Jiang, L. Ultraefast fabrication of colloidal photonic crystals by spray coating. Macromol. Rapid. Commun. 2009, 30, 598-603. [CrossRef] [PubMed]

17. Hou, S.Y.; Xie, A.Z.; Xie, Z.W.; Tobing, L.Y.M.; Zhou, J.; Tjahjana, L.; Yu, J.H.; Hettiarachchi, C.; Zhang, D.H.; Dang, C.; et al. Concurrent inhibition and redistribution of spontaneous emission from all inorganic perovskite photonic crystals. ACS Photonics 2019, 6, 1331-1337. [CrossRef]

18. Chen, C.; Zhu, Z.G.; Shih, W.H.; Ge, Q.Q.; Liu, M.J.; Zhu, X.R. Facile preparation and self assembly of monodisperse polystyrene nanospheres for photonic crystals. J. Nanosci. Nanotechnol. 2015, 15, 3239-3243. [CrossRef] [PubMed]

19. Vogel, N.; Retsch, M.; Fustin, C.; del Campo, A.; Jonas, U. Advances in colloidal assembly: The design of structure and hierarchy in two and three dimensions. Chem. Rev. 2015, 115, 6265-6311. [CrossRef] [PubMed]

20. Waterhouse, G.I.N.; Chen, W.T.; Chan, A.; Sun-waterhouse, D.X. Achieving color and function with structure: Optical and catalytic support properties of $\mathrm{zro}_{2}$ inverse opal thin films. ACS Omega 2018, 3, 9658-9674. [CrossRef] [PubMed]

21. Jiang, P.; Bertone, J.F.; Hwang, K.S.; Colvin, V.L. Single-crystal colloidal multilayers of controlled thickness. Chem. Mater. 1999, 11, 2132-2140.

22. Hufziger, K.T.; Zrimsek, A.B.; Asher, S.A. Solid Deep Ultraviolet Diffracting Inverse Opal Photonic Crystals. ACS Appl. Nano Mater. 2018, 1, 7016-7024. [CrossRef]

23. Wang, J.X.; Liang, J.; Wu, H.M.; Yuan, W.F.; Wen, Y.Q.; Song, Y.L.; Jiang, L. A facile method of shielding from UV damage by polymer photonic crystals. Polym. Int. 2008, 57, 509-514. [CrossRef]

(C) 2020 by the authors. Licensee MDPI, Basel, Switzerland. This article is an open access article distributed under the terms and conditions of the Creative Commons Attribution (CC BY) license (http://creativecommons.org/licenses/by/4.0/). 